\title{
Estudo de antecedentes da aceitação de sites de reservas online, intenção de compra e boca a boca positivo
}

\author{
Study of antecedents of acceptance of online booking sites, purchase intention and positive \\ word of mouth
}

\section{Estudio de antecedentes de aceptación de sitios de reserva en línea, intención de compra y boca a boca positiva}

Robledo Cunha Rubem ${ }^{1}$

Resumo: Este estudo buscou compreender os fatores que influenciam a intenção de compra e o boca a boca positivo de sites de reserva online, sendo testadas as relações entre a comunicabilidade, complexidade, vantagem relativa e risco percebido sobre atitude, influenciando a intenção de compra e o boca a boca positivo. O modelo foi testado por meio de equações estruturais, com dados coletados por meio de um questionário aplicado, de forma online, em 453 respondentes para a avaliação de cada construto. Os resultados obtidos confirmam a confiança do modelo e as hipóteses apresentadas, com um bom nível de explicação das variáveis dependentes. Esse estudo apresenta elementos que podem ser trabalhados pelas empresas que visam impulsionar a utilização e propagação dos sites de reservas, assim como pontos de atenção a serem considerados pelos concorrentes.

Palavras-chave: Boca a boca. Comércio eletrônico. Intenção de compra. Sites de reservas. Turismo.

Abstract: This study sought to understand the factors that influence the purchase intention and the positive wordof-mouth of online booking sites, testing the relationships between communicability, complexity, relative advantage and perceived risk on attitude, influencing the purchase intention and positive word-of-mouth. The model was tested using structural equations, with data collected through a questionnaire applied online to 453 respondents for the assessment of each construct. The results obtained confirm the model's confidence and the hypotheses presented, with a good level of explanation of the dependent variables. The study presents elements that can be worked on by companies that aim to boost the use and spread of booking sites, as well as points of attention to be considered by competitors.

Key words: Mouth to mouth. E-commerce. Buy intention. Booking sites. Tourism.

Resumen: Este estudio buscó comprender los factores que influyen en la intención de compra y el boca a boca positivo de los sitios de reserva en línea, probando las relaciones entre comunicabilidad, complejidad, ventaja relativa y riesgo percibido en la actitud, influyendo en la intención de compra y boca a boca positivo. El modelo se probó utilizando ecuaciones estructurales, con los datos recopilados a través de un cuestionario aplicado en línea a 453 encuestados para la evaluación de cada constructo. Los resultados obtenidos confirman la confianza del modelo y las hipótesis presentadas, con un buen nivel de explicación de las variables dependientes. El estudio presenta elementos en los que las empresas pueden trabajar para mejorar el uso y la difusión de los sitios de reserva, así como puntos de atención a ser considerados por los competidores.

Palabras Clave: Boca a boca. Comercio electrónico. Intención de compra. Sitios de reserva. Turismo.

\footnotetext{
${ }^{1}$ Formação/curso: Mestre em Administração de Empresas com ênfase em Marketing. Instituição: PUC Rio - IAG, Rio de Janeiro - RJ, Brasil E-mail: robledo.rubem@uol.com.br

${ }^{2}$ Formação/curso: Doutor em Administração de Empresas com ênfase em Marketing. Instituição: PUC Rio - IAG, Rio de Janeiro - RJ, Brasil). E-mail: jorge.brantes@gmail.com

${ }^{3}$ Formação/curso: Doutor em Administração. Instituição: Universidade Federal Fluminense (UFF), Niterói - RJ, Brasil. E-mail: robertopqfalcao@gmail.com
} 
ESTUDO DE ANTECEDENTES DA ACEITAÇÃO

DE SITES DE RESERVAS ONLINE, INTENÇÃO

DE COMPRA E BOCA A BOCA POSITIVO
Robledo Cunha Rubem

Jorge Brantes Ferreira

Roberto Pessoa de Queiroz Falcão

\section{Introdução}

O turismo foi um setor profundamente afetado pelo advento da internet e do e-commerce (BUHALIS; LAW, 2008). A era do turismo digital e do uso de smartphones em viagem trouxe tecnologias, tais como a inteligência artificial e os algoritmos, o uso de bots, experiência de compra personalizada, mídias sociais, blogs e sistema de reserva automatizada, que transformam a cada dia a experiência do turista (LAW; CHAN; WANG, 2018; ADEOLA; EVANS, 2019; MELIÁN-ALZOLA; FERNÁNDEZ-MONROY; HIDALGO-PEÑATE, 2020).

$\mathrm{O}$ uso da internet traz benefícios para o planejamento e a execução de viagens, devido à fácil obtenção de informações sobre as características do destino (HO; LIN; CHEN, 2012). Contudo, além dos consumidores, as empresas que atuam neste setor também foram beneficiadas com o desenvolvimento tecnológico. Destacam-se os sistemas de reserva e distribuição global (GDS), que surgiram nos anos 70 (GRETZEL; FESENMAIER, 2009) e o mais recente advento das agências online que intermediam passagens aéreas, hospedagens, reservas de carros, e ingressos para atrações (BUHALIS; LAW, 2008; JENG; LO, 2019).

Opiniões de terceiros também são extremamente importantes no processo decisório de qualquer viajante (LÓPEZ; SICILIA, 2014; LUO; ZHONG, 2015), podendo estar relacionadas à escolha do canal para realizar reservas e à escolha do destino. Opiniões de familiares, amigos, colegas de trabalho, ou até mesmo do (a) recepcionista do hotel são úteis tanto no ato do planejamento quanto para decisões de compra durante viagens (GRETZEL; FESENMAIER, 2009).

Nesse sentido, o consumidor torna-se cada vez mais protagonista na compra online de turismo, seja como iniciador, comprador ou influenciador da viagem de outras pessoas, fornecendo dicas ou sugerindo um site de reserva online. De fato, observa-se que os turistas têm sido bombardeados pelas mais diversas fontes de informações de referência on-line, presentes nas redes sociais digitais (LEUNG et al., 2013). Esse tipo de informação é frequentemente usado em suas decisões de compra, o chamado "boca a boca eletrônico" ou "eWOM" (LIU; PARK, 2015), a exemplo dos sites como TripAdvisor, Hostelworld e Booking.com. Neles, além de obter informações sobre a qualidade do serviço, fazer contatos com fornecedores relacionados ao turismo, pode-se realizar reservas. A literatura existente explora os impactos do eWOM no turismo e na hospitalidade, incluindo aspectos que motivam os consumidores a compartilhar suas opiniões sobre negócios de viagens (HENNIG-THURAU et al., 2004; GODES; MAYZLIN, 2004). 
ESTUDO DE ANTECEDENTES DA ACEITAÇÃO

DE SITES DE RESERVAS ONLINE, INTENÇÃ̃

DE COMPRA E BOCA A BOCA POSITIVO
Robledo Cunha Rubem Jorge Brantes Ferreira Roberto Pessoa de Queiroz Falcão

Vale ressaltar que o crescimento na compra de viagens pela internet tem sido empurrado pelas agências de viagens online (OTA) que representavam 56\% de participação nas vendas em 2017, ou 17,4 bilhões de reais (EUROMONITOR INTERNATIONAL, 2017). Em 2020, antes da pandemia do COVID19, previa-se que as vendas on-line de viagens representariam 52\% globalmente até 2024, sendo as vendas via celular representando um quarto de todas as reservas de viagens em termos de valor. Segundo o relatório, as companhias aéreas lideram o caminho, com quase $70 \%$ das vendas feitas online, em comparação com $47 \%$ de hospedagem e atividades e experiências atrás dos $12 \%$ em 2019. (EUROMONITOR INTERNATIONAL, 2019). O Booking.com, Hoteis.com, Decolar.com e Expedia, são alguns exemplos dos que atuam no Brasil, tendo a líder Decolar, tendo atingido $\mathrm{R} \$ 8$ bilhões em vendas neste mesmo ano. No outro extremo estão as agências de viagens tradicionais, extremamente impactadas pelo crescimento das transações online e do acesso à rede. O consumidor empoderado, agora não tem mais necessidade de utilizar agentes de viagem presenciais, nem tampouco necessita pagar por essa intermediação. Portanto, entender o que leva as pessoas a comprarem e recomendarem os agentes de viagem online é o objetivo deste estudo. Surgem indagações como: "Por que comprar por meio da Decolar.com uma passagem que pode ser adquirida no próprio site da Gol?”, ou “ Por que reservar pelo Booking um quarto de um hotel Ibis, e não pelo site do hotel?", ou "Por que recomendar o uso de um site de reserva?"

Para este estudo, foi analisado o comportamento de consumidores, buscando entender a utilização dos sites de reservas. Pesquisas já foram realizadas nesse sentido, a exemplo da que avalia a intenção de compra de viagens pela internet (AMARO; DUARTE, 2015), a que estuda a influência do conteúdo gerado pelo usuário (CGU) como fator decisório no processo de compra de produtos e serviços turísticos via Internet, usando a Teoria do Comportamento Planejado (SILVA; MENDES-FILHO; CORREA, 2017), e da que analisa os antecedentes da intenção do uso de comentários de viagem online na escolha de um alojamento, usando o Modelo de Aceitação Tecnológica, a Teoria Motivacional, da Similaridade e da Confiabilidade (SILVA; MENDES FILHO; MARQUES JÚNIOR, 2019). Contudo, esse tipo de avaliação mais geral acaba agrupando, na mesma análise, aquisições feitas por meio dos sites de reservas online e também por meio dos próprios sites das empresas, sem elucidar particularidades e diferenças entre os dois tipos de consumo.

\section{Revisão de Literatura}

\subsection{Tecnologia e turismo e o boca a boca eletrônico}


ESTUDO DE ANTECEDENTES DA ACEITAÇÃO

DE SITES DE RESERVAS ONLINE, INTENÇÃO

DE COMPRA E BOCA A BOCA POSITIVO
Robledo Cunha Rubem Jorge Brantes Ferreira Roberto Pessoa de Queiroz Falcão

A internet transformou drasticamente a forma como as pessoas consomem turismo (GRETZEL; FESENMAIER, 2009). Em alguns casos, todo planejamento de uma viagem é realizado por meio da internet, desde a busca por informações e de opiniões de outras pessoas passando pelas escolhas do destino, acomodações e passagens (LITVIN; GOLDSMITH; PAN, 2008) e reservas (AGAG; ELMASRY, 2016b; AMARO; DUARTE, 2015), o que mostra a consolidação do uso de internet para o turismo (YU, 2008; ALMEIDA; ALMEIDA; MOTA, 2019).

Nesse sentido, o e-commerce não somente abriu um novo canal de distribuição, mas criou um novo mercado (WU; CHANG, 2006). E muitas são as empresas cuja atuação é 100\% online, sendo tais ofertas não disponibilizadas em outros canais, ou seja, o cliente só pode adquirir de forma online. No caso do turismo, um exemplo dessas empresas são os sites de reservas online (CASTILLO-MANZANO; LÓPEZ-VALPUESTA, 2010).

De acordo com a definição de Litvin, Goldsmith e Pan (2008), o boca a boca é uma comunicação entre consumidores sobre um produto, serviço ou uma empresa, onde as fontes são consideradas independentes de influências comerciais. Melhor dizendo, seria a comunicação realizada entre consumidores, de modo informal, a respeito do uso, posse e características de determinados produtos, e de seus vendedores (WESTBROOK, 1987).

O boca a boca eletrônico oferece vantagens sobre o tradicional, como maior conveniência, anonimato, oportunidade de se comunicar com muitos indivíduos simultaneamente e ausência de restrições em relação ao tempo e ao espaço (LITVIN; GOLDSMITH; PAN, 2008). Assim, pode-se dizer que o boca a boca eletrônico exerce uma influência mais ampla do que o tradicional (PAN; ZHANG, 2011).

Uma forma de minimizar os riscos emocionais ligados à intangibilidade dos serviços turísticos, como a qualidade do quarto de hotel ou conforto do transporte, seria a análise de comentários expressos por outras pessoas (LUO; ZHONG, 2015). Os consumidores valem-se desses comentários, estrelas, notas, e rankings para analisarem produtos e serviços oferecidos nos ambientes online. Os números de palavras totais e negativas também exercem influência no momento de avaliação das alternativas (BAEK; AHN; CHOI, 2012).

Ladhari e Michaud (2015) evidenciaram, em suas pesquisas, que quanto maior for o boca a boca eletrônico positivo a respeito de um determinado hotel, melhor será também a atitude, a confiança, a percepção de qualidade e a intenção de reserva por parte dos consumidores. Assim, Vermeulen e Seegers (2009) indicam que as avaliações positivas de clientes melhoram a percepção dos hotéis entre potenciais consumidores. Dickinger e Mazanec (2008) também revelam que as recomendações de amigos e avaliações online são os fatores mais influenciadores de reservas de hotéis pela internet, corroborando 
com Ye et al. (2011) e Litvin, Goldsmith e Pan (2008). Buscar indicações e conselhos é algo tão importante no planejamento de uma viagem que algumas empresas criaram comunidades online com o objetivo de facilitar essa tarefa aos consumidores (QU; LEE, 2011), onde acessam opiniões até de pessoas desconhecidas que já tiveram experiências próximas das que se pretende. Nesse sentido, Casaló, Flavián e Guinalíu (2010) e Wang e Fesenmaier (2004) atestam a importância da facilidade de uso e a utilidade percebida, e de benefícios hedônicos e normativos para participação nessas comunidades.

$\mathrm{Na}$ literatura acadêmica nacional, destacam-se os trabalhos que estudaram a influência do conteúdo gerado pelo usuário (CGU) como fator decisório no processo de compra de produtos e serviços turísticos via Internet, usando a Teoria do Comportamento Planejado (SILVA; MENDES-FILHO, CORREA, 2017). Outro estudo recente e relevante é o que analisa os antecedentes da intenção do uso de comentários de viagem on-line na escolha de um alojamento, usando o Modelo de Aceitação Tecnológica, a Teoria Motivacional, da Similaridade e da Confiabilidade (SILVA; MENDES FILHO; MARQUES JÚNIOR, 2019). Ambos evidenciaram a importância dos comentários on-line ao influenciarem a intenção de escolha de um destino turístico ou meio de hospedagem.

\subsection{Atitude em relação a intenção de compra e o boca a boca positivo}

A atitude é um conjunto de julgamentos cognitivos e afetivos de um indivíduo com relação a uma determinada ação ou comportamento, sendo apresentada como um construto fundamental para formação da intenção de compra no meio online (INGHAM; CADIEUX; MEKKI BERRADA, 2015; VIJAYASARATHY, 2004). Diversos estudos relacionam atitude e a intenção de compra de viagens online (reservas de hotéis, passagens e passeios) de forma positiva (AGAG; EL-MASRY, 2016a, 2016b; LEE; QU; KIM, 2007). Sendo assim, a atitude destaca-se como o elemento que gera maior impacto na intenção de compra (BIGNÉ et al., 2010; MOROSAN; JEONG, 2008; AMARO; DUARTE, 2015) e fator determinante para a intenção de uso de sites de reservas online. Entende-se, portanto, que a atitude deve ser considerada como antecedente da intenção de uso de sites de reservas online, assim propõe-se:

H1: A atitude em relação aos sites de reservas online possui efeito direto e positivo sobre a intenção de compra por meio de sites de reservas online.

Bem como ocorre para a intenção de compra, a atitude também é percebida como um construto relevante na formação do boca a boca positivo. Essa relação é importante, pois uma atitude favorável pode levar a pessoa não somente a adquirir algo, mas também a fazer recomendações. Um motivo que pode explicar tal comportamento é a predominância de aspectos afetivos, em relação aos cognitivos na influência da atitude sobre o boca a boca (SHIH; LAI; CHENG, 2013), o que é corroborado por outros 
estudos que também encontraram um efeito significativo desse construto sobre o boca a boca positivo (AGAG; EL-MASRY, 2016b; LIEN; CAO, 2014). Por conseguinte, a atitude vem sendo apontada como um elemento que impacta diretamente o boca a boca positivo dos consumidores. Posto isso, espera-se que:

H2: A atitude em relação aos sites de reservas online possui efeito direto e positivo sobre o boca a boca positivo a respeito dos sites de reservas online.

\subsection{Comunicabilidade}

De acordo com a Teoria da Ação Racionalizada, um comportamento não é resultado somente da atitude em relação ao mesmo, mas também de normas subjetivas, oriundas das pressões sociais sofridas pelas pessoas (FISHBEIN; AJZEN, 1975). E segundo Burnkrant e Cousineau (1975), um dos fatores que influenciam mais profundamente o comportamento de um indivíduo é a influência de outros.

Entretanto, as normas subjetivas não têm apresentado a mesma influência exercida sobre certos comportamentos, quando analisada em relação a intenção de compras online (PAVLOU; FYGENSON, 2006; WANG et al., 2007; SAN MARTÍN; HERRERO, 2012). Considerando a importância da influência de terceiros e que normas subjetivas não explicam bem a intenção de compras online, Amaro e Duarte (2015) utilizaram a Comunicabilidade, uma forma diferente de influência social, relacionada à influência que pode ser exercida por amigos e familiares, dado que as pessoas tendem a encarar um determinado tipo de comportamento com maior naturalidade quando sabem que o mesmo já é praticado por pessoas próximas (MORRISON et al., 2001). Morrison et al. (2001) identificaram que as pessoas estão mais pré-dispostas a fazer reservas online, sobretudo se souberem que outros o estão fazendo. Entretanto, Amaro e Duarte (2015) não buscaram diferenciar sites de reservas de agentes de viagem dos sites dos próprios fornecedores, dando margem a um resultado diferente no contexto de sites de reservas online. Essa sugestão se justifica, pois, a natureza do serviço de sites de reservas é diferente dos sites dos próprios fornecedores, já que sites de reservas funcionam como intermediários para o consumidor. Dado que a atitude já foi apresentada como um construto fundamental para formação da intenção de compra no meio online, (INGHAM; CADIEUX; MEKKI BERRADA, 2015; VIJAYASARATHY, 2004), podendo haver uma relação mais significativa da comunicabilidade com a atitude, do que diretamente com a intenção de compra, propõe-se:

\section{H3: A comunicabilidade possui efeito direto e positivo sobre a atitude relacionada a sites de} reservas online. 
ESTUDO DE ANTECEDENTES DA ACEITAÇÃO

DE SITES DE RESERVAS ONLINE, INTENÇÃO

DE COMPRA E BOCA A BOCA POSITIVO
Robledo Cunha Rubem

Jorge Brantes Ferreira

Roberto Pessoa de Queiroz Falcão

\subsection{Teoria da difusão de inovações e modelo de aceitação de tecnologias}

A teoria seminal da difusão de inovações, testada em diversos contextos, permanece sendo a mais utilizada quando se deseja entender a adoção de uma nova tecnologia (ROGERS, 2003). Cinco atributos são elencados para avaliação do potencial de adoção ou difusão de uma determinada inovação: complexidade, compatibilidade, vantagem relativa, observabilidade e testabilidade. Já o modelo de aceitação de tecnologias (TAM) baseia-se em dois fatores cognitivos para explicar o nível da adoção de uma nova tecnologia: a facilidade de uso e a utilidade percebida (BAGOZZI; DAVIS; WARSHAW, 1992; VENKATESH et al., 2003). Por terem elementos considerados equivalentes, toma-se como base para este estudo, a teoria de difusão de inovações. A percepção de complexidade é similar à facilidade de uso (TAM), segundo Wu e Wang (2005) e Amaro e Duarte (2015). Da mesma forma, utilidade e vantagens relativas são construtos semelhantes, o que é corroborado também por outros autores (RIEMENSCHNEIDER; HARDGRAVE; DAVIS, 2002; WU; WANG, 2005).

Devido a falta de um suporte teórico consistente, a testabilidade e a observabilidade (Rogers, 2003) não serão tratadas neste estudo (AGAG \& EL-MASRY, 2016a; AMARO; DUARTE, 2015). Da mesma forma, a compatibilidade também não será verificada por estarmos tratando de um serviço que pode ser entendido como tendo natureza híbrida, dado que a aquisição de viagens online surgiu a partir da propagação da internet. Todavia, a utilização de intermediários para as compras de viagens é muito anterior ao surgimento da internet. Portanto seria difícil especificar na pesquisa a que a compatibilidade estaria se referindo.

\subsection{Complexidade}

O nível de dificuldade enfrentado por uma pessoa para aprender e utilizar uma determinada tecnologia é representado pela percepção de complexidade (ROGERS, 2003). À medida que pessoas enxergam uma tecnologia como simples e fácil de usar, a mesma possui uma probabilidade maior de aceitação e um número potencial maior de usuários (DAVIS; BAGOZZI; WARSHAW, 1989). Conforme sustenta o modelo TAM, a facilidade de uso é um fator determinante para a construção da atitude (DAVIS, 1989). Ao considerar somente o segmento turístico, muitos estudos confirmam a influência da percepção de complexidade sobre a atitude, seja no contexto de sites de reserva de hotéis (AGAG; EL-MASRY, 2016b; MOROSAN; JEONG, 2008), na compra de viagens online (AMARO; 
DUARTE, 2015), na participação em comunidades viagens online (AGAG; EL-MASRY, 2016a) e em relação à agências de viagens online (CHO; AGRUSA, 2006). Dessa forma, podemos considerar que:

H4: A percepção de complexidade possui efeito direto e negativo sobre a atitude relacionada a sites de reservas online.

\subsection{Vantagem relativa}

Como mencionado anteriormente, o conceito de vantagem relativa da teoria de difusão de inovações é semelhante a utilidade percebida do modelo de aceitação de tecnologias (AMARO; DUARTE, 2015; RIEMENSCHNEIDER; HARDGRAVE; DAVIS, 2002; WU; WANG, 2005). Contudo, existe uma diferença entre as duas, que vale ser ressaltada para o nosso estudo. Quando falamos de vantagem relativa, podemos atribuir a esse construto a característica de comparação entre o objeto inovador em relação aos seus antecessores, sendo que não podemos fazer o mesmo quando estamos tratando do construto utilidade percebida (KARAHANNA et al., 2002). Portanto, podemos levar em conta que os benefícios creditados aos sites de reservas online podem ser comparados às opções anteriores que os clientes possuíam. No caso desse estudo, as alternativas seriam a aquisição de viagens por meio de agências de viagens tradicionais (AMARO; DUARTE, 2015), e a reserva de hotéis ou passagens aéreas em seus próprios sites.

Dessa forma, considerando a indicação do TAM de que a utilidade percebida contribui para formação de uma atitude favorável (BAGOZZI; DAVIS; WARSHAW, 1992) e a indicação de que utilidade e vantagem relativa tratam de aspectos similares de uma inovação (AMARO; DUARTE, 2015; RIEMENSCHNEIDER; HARDGRAVE; DAVIS, 2002; WU; WANG, 2005), propõe-se:

H5: A vantagem relativa percebida possui efeito direto e positivo sobre a atitude relacionada a sites de reservas online.

\subsection{Risco percebido}

No caso das agências de viagens tradicionais, a figura do agente transmite uma segurança maior, posto que o consumidor estabelece uma relação de confiança com este profissional e acredita na sua experiência no ramo para programar as suas viagens (CHEYNE; DOWNES; LEGG, 2006). Considerando que, por meio dos sites de reservas, não existe essa relação pessoal com a figura de um profissional auxiliando na programação da viagem, é possível que o risco percebido em relação a aquisição por este canal seja maior, visto que os produtos de viagens são caracterizados pela 
intangibilidade, e, portanto, enxerga-se um risco maior do que em um produto tangível, como um computador, por exemplo (LIN; JONES; WESTWOOD, 2009). O trabalho seminal de Jarvenpaa e Todd (1997) já sugeria uma série de riscos que poderiam estar associados às compras online, como riscos econômicos, riscos de performance ou funcionamento, riscos de segurança e também de privacidade. $\mathrm{O}$ risco percebido está relacionado negativamente à intenção de compra de passagens aéreas (KIM; QU; KIM, 2009; KIM; KIM; LEONG, 2005; KOLSAKER; LEEKELLEY; CHOY, 2004), e de compras de viagens online no geral (JENSEN, 2012). O mesmo tipo de relação também foi encontrado entre risco percebido e atitude (BIGNÉ et al., 2010). Logo, propõe-se:

H6: $O$ risco percebido possui efeito direto e negativo sobre a atitude relacionada a sites de reservas online.

Com base nas hipóteses construídas em cima do referencial teórico, o modelo proposto apresenta-se na Figura 1.

Figura 1. Modelo proposto

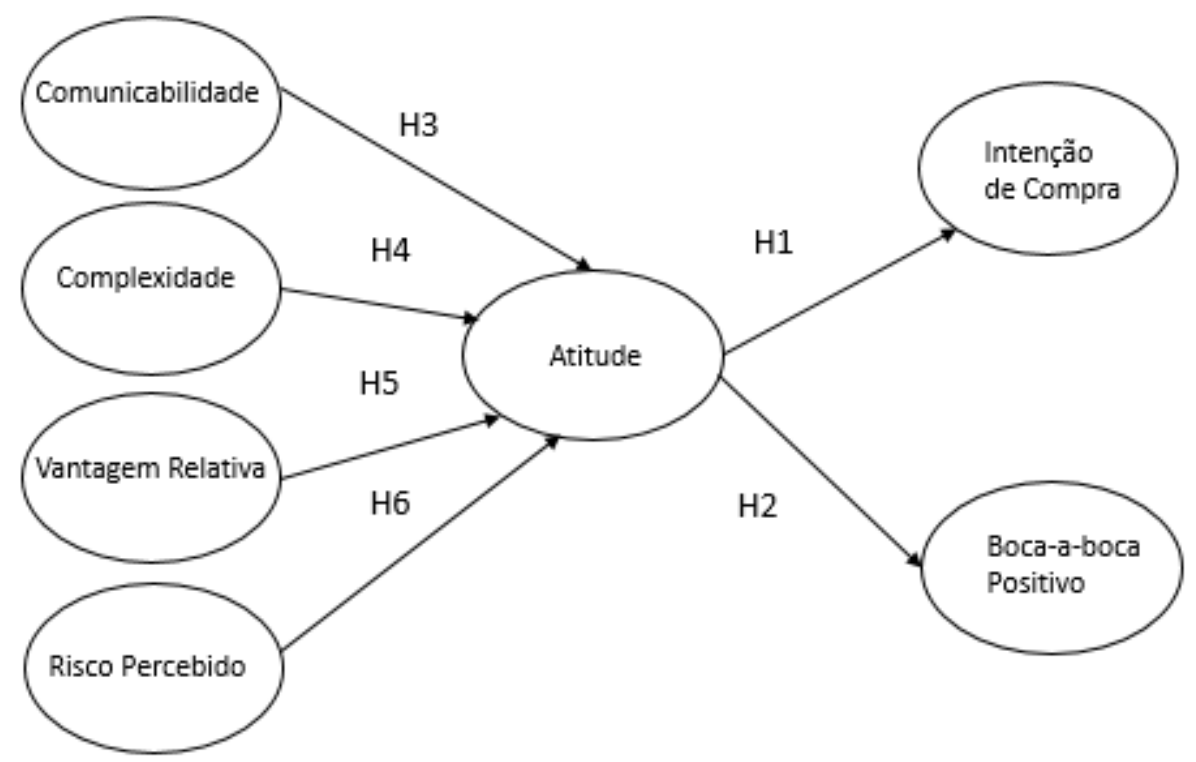

Fonte: Elaboração própria.

\section{Método}

Com o objetivo de realizar o teste das hipóteses formuladas para o estudo, foi realizada uma survey com uma amostra não-probabilística da população de interesse (AAKER et al., 2012). Ressaltase que, para o problema de pesquisa estudado, já existe um corpo razoável de artigos acadêmicos relacionados, inclusive com estudos que definem modelos para estudo das variáveis em questão e 
utilizam metodologia quantitativa idêntica a aplicada neste trabalho (AMARO; DUARTE, 2015; AGAG; EL-MASRY, 2016a; SILVA; MENDES-FILHO, CORREA, 2017; SILVA; MENDES FILHO; MARQUES JÚNIOR, 2019). A análise de dados foi feita por meio de modelagem de equações estruturais (MEE), utilizando o software AMOS. O uso de MEE é adequado às questões desta pesquisa e aos testes exigidos pelas hipóteses formuladas, uma vez que permite a estimação de relações simultâneas entre múltiplas variáveis independentes e dependentes (HAIR et al., 2009). Com a utilização desta técnica, é possível a avaliação conjunta dos efeitos de todos os construtos envolvidos no modelo proposto, evitando possíveis distorções que ocorreriam nas dependências observadas entre as variáveis, caso fossem estudadas separadamente (HAIR et al., 2009).

\subsection{Amostra e procedimentos de coleta de dados}

A população de interesse da pesquisa é composta por jovens e adultos brasileiros que já fizeram algum tipo de viagem envolvendo reservas de hotel, passagem aérea, passeios, etc. A amostra do estudo foi composta de respondentes brasileiros de diferentes regiões do país, sendo o questionário online criado na ferramenta Qualtrics e encaminhado por meio de redes sociais (Facebook e Whatsapp) para os respondentes. As respostas foram coletadas entre os dias 04/01/2018 e 28/01/2018.

Ao todo, foram registradas 591 respostas, sendo descartadas 138 que não preencheram o questionário até o final. A amostra final foi composta de 453 respondentes (43,30\% homens e 56,70\% mulheres, cuja faixa etária variou entre 20 a 60 anos). A renda familiar dos respondentes dividiu-se em acima de 10.000 reais $(29,24 \%)$, entre 6.000 e 10.000 reais $(27,46 \%)$ e entre 3.500 e 6.000 reais $(21,43 \%)$. Quanto ao perfil turístico dos respondentes, parte alegou não viajar todos os anos $(27,46 \%)$, seguido por aqueles que afirmaram viajar somente uma vez $(23,21 \%)$ ou duas vezes $(22,54 \%)$ por ano. A grande maioria dos participantes respondeu que o motivo principal de suas viagens era o lazer (76,34\%) e mais de 50\% informou já ter viajado ao exterior. Em relação à utilização de sites de reservas, duas marcas destacaram-se como mais utilizadas: Booking.com (224 citações ou 29,20\%), e Decolar.com (184 citações ou 23,99\%). Já dos tipos de compra realizada nesses sites, a reserva de hotel foi a mais citada 280 vezes $(40,82 \%)$.

$\mathrm{O}$ instrumento de coleta de dados foi composto de 44 perguntas, sendo 35 delas relacionadas aos construtos testados no modelo - intenção de compra ( 3 itens), boca a boca positivo (3 itens), atitude (5 itens), comunicabilidade (3 itens), complexidade (4 itens), vantagem relativa (4 itens) e risco percebido (5 itens) - e ao perfil do respondente em relação a serviços turísticos (5 itens) e aos dados demográficos (4 itens). Foram dispostas primeiramente perguntas referentes ao consumo de serviços 
turísticos, e em seguida as relacionadas às hipóteses e construtos, terminando pelos dados demográficos. Alguns exemplos de marcas de sites de reservas online foram ilustrados no início do questionário para que os respondentes vislumbrassem o que estava sendo pesquisado. Todas as escalas utilizadas originalmente obtidas em língua inglesa, foram traduzidas e adaptadas para língua portuguesa (ver BRISLIN, 1970; SPERBER, 2004). Foram realizados pré-testes escrito e online, respectivamente com 10 e 12 respondentes, buscando-se ajustar a fluidez e clareza do questionário. Os dados coletados no software Qualtrics foram posteriormente processados estatisticamente por meio do SPSS 21 e AMOS 21.

\section{Resultados}

\subsection{Teste de variância comum do método}

Dado que a variância comum do método (CMV) pode gerar problemas, considerando que todos itens, variáveis dependentes e independentes, foram coletadas opiniões dos mesmos informantes, e em seguida, foi feito o teste de um fator de Harman (PODSAKOFF; ORGAN, 1986), a fim de examinar até que ponto esse viés estava presente nos dados da pesquisa. Os resultados da análise de componentes principais indicaram a presença de sete fatores com valor próprio superior a 1 . Ao mesmo tempo, nenhum dos fatores representava quase toda a variação (o fator que explicava os mais conseguiram capturar apenas $36.1 \%$ da variação total).

\subsection{Avaliação do modelo de mensuração}

No sentido de verificar a validade, unidimensionalidade e confiabilidade das escalas utilizadas, foi feita uma análise fatorial confirmatória (CFA), permitindo avaliar o quanto cada item medido se relaciona com cada construto particularmente e verificar os diferentes índices de ajustes incrementais e absolutos para o ajuste do modelo.

O modelo foi testado considerando todos 27 indicadores correspondentes aos construtos propostos, no entanto os índices de ajuste não apresentaram resultados recomendados pela literatura, sendo necessário um refinamento do modelo, extraindo-se os itens COM3 (Comunicabilidade), CPL2 (Complexidade) e VAT1 (Vantagem Relativa). Com a retirada desses três itens, o modelo final passou a conter 24 indicadores com os seguintes índices de ajuste (Tabela 1): RMSEA $=0,042 ; \mathrm{CI}=0,035$ até 0,049; CFI = 0,971; IFI $=0,972 ;$ TLI $=0,963 ;$ X2 $=387,435 ;$ d.f. $=217, \mathrm{p}<0,001$, X2/d.f. $=1,785$. Os 
resultados de todos esses índices indicam que o modelo final de mensuração possui um ajuste satisfatório (HU; BENTLER, 1999; SCHREIBER et al., 2006).

Tabela 1. Índices de Ajuste do Modelo de Mensuração

\begin{tabular}{c|c}
\hline Índice de Ajuste & Modelo Proposto \\
\hline$\chi 2 /$ c.f & 1,785 \\
\hline CFI & 0,971 \\
\hline TLI & 0,963 \\
\hline IFI & 0,972 \\
\hline RMSEA & 0,042 \\
\hline
\end{tabular}

Fonte: Dados da pesquisa.

A validade de face para todas as escalas foi garantida pela escolha das já utilizadas na literatura, tradução criteriosa e pré-testes durante o desenvolvimento do instrumento de pesquisa. Já para validade nomológica foi analisada a matriz de correlação entre construtos, sendo todas as correlações significativas e estando na direção esperada. No que diz respeito à validade convergente, foi calculada a variância extraída média para cada construto (AVE), sendo todos os valores entre 0,50 e 0,67, evidenciando a validade convergente das escalas utilizadas. Foram também examinadas as cargas fatoriais padronizadas para cada variável observável nos construtos com suas respectivas significâncias. Quanto maiores forem essas cargas, mais fortes são as evidências de que as variáveis medidas representam os construtos subjacentes aos quais estão associadas, indicando validade convergente e unidimensionalidade. A Tabela 2 apresenta as cargas fatoriais padronizadas e suas significâncias para cada indicador presente no modelo de mensuração estimado, todas significativas e acima de 0,5 (HAIR et al., 2009). As escalas utilizadas também atenderam aos níveis mínimos de confiabilidade e consistência interna considerados adequados (FORNELL; LARCKER, 1981), apresentando valores entre 0,62 e 0,86 para o coeficiente alfa e entre 0,70 e 0,87 para a confiabilidade composta. Por fim, todas as variâncias compartilhadas foram inferiores à variância extraída pelos itens que medem os construtos, indicando validade discriminante adequada.

Tabela 2. Cargas fatoriais padronizadas e significância por indicador

\begin{tabular}{c|c|c|c}
\hline \multirow{2}{*}{ Construto } & Indicador & $\begin{array}{c}\text { Carga } \\
\text { Padronizada }\end{array}$ & Significância \\
\hline \multirow{4}{*}{ Risco percebido } & RIS1 & 0.532 & $<0,001$ \\
\cline { 2 - 4 } & RIS2 & 0.546 & $<0,001$ \\
\cline { 2 - 4 } & RIS3 & 0.877 & $<0,001$ \\
\cline { 2 - 4 } & RIS4 & 0.803 & $<0,001$ \\
\cline { 2 - 4 } Comunicabilidade & RIS5 & 0.538 & $<0,001$ \\
\cline { 2 - 4 } & COM1 & 0.668 & $<0,001$ \\
\hline \multirow{3}{*}{ Complexidade } & COM2 & 0.714 & $<0,001$ \\
\cline { 2 - 4 } & CPL1 & 0.519 & $<0,001$ \\
\cline { 2 - 4 } & CPL3 & 0.776 & $<0,001$ \\
\hline
\end{tabular}

Observatório de Inovação do Turismo - Revista Acadêmica

Vol. XIX, n 3, dezembro - 2020 


\begin{tabular}{c|c|c|c}
\hline \multirow{4}{*}{ Vantagem relativa } & VAT2 & 0.585 & $<0,001$ \\
\cline { 2 - 4 } & VAT3 & 0.759 & $<0,001$ \\
\cline { 2 - 4 } & VAT4 & 0.587 & $<0,001$ \\
\hline \multirow{3}{*}{ Atitude } & ATI1 & 0.688 & $<0,001$ \\
\cline { 2 - 4 } & ATI2 & 0.751 & $<0,001$ \\
\cline { 2 - 4 } & ATI3 & 0.811 & $<0,001$ \\
\cline { 2 - 4 } & ATI4 & 0.748 & $<0,001$ \\
\hline \multirow{3}{*}{ Boca a boca positivo } & ATI5 & 0.759 & $<0,001$ \\
\cline { 2 - 4 } & BOC1 & 0.889 & $<0,001$ \\
\cline { 2 - 4 } & BOC2 & 0.700 & $<0,001$ \\
\hline \multirow{3}{*}{ Intenção de compra } & BOC3 & 0.835 & $<0,001$ \\
\cline { 2 - 4 } & INT1 & 0.828 & $<0,001$ \\
\cline { 2 - 4 } & INT2 & 0.837 & 0.782 \\
\hline
\end{tabular}

Fonte: Dados da pesquisa.

\subsection{Modelagem estrutural}

A modelagem de equações estruturais (SEM) foi utilizada para testar o modelo proposto e as hipóteses da pesquisa (HAIR et al., 2009). Todos os índices indicaram um bom ajuste do modelo aos dados, conforme ilustrado na Tabela 3.

Tabela 3. Índices de ajuste do modelo estrutural

\begin{tabular}{c|c}
\hline Índice de Ajuste & Modelo Proposto \\
\hline$\chi 2 /$ c.f & 3,642 \\
\hline CFI & 0,903 \\
\hline TLI & 0,901 \\
\hline IFI & 0,904 \\
\hline RMSEA & 0,077 \\
\hline
\end{tabular}

Fonte: Dados da pesquisa.

A estatística qui-quadrada obtida para o modelo foi significativa estatisticamente $(\chi 2=830,432$, d.f. $=228, p<0,001)$. Já a razão $\chi 2 /$ c.f. foi de 3,642, um pouco superior ao que é recomendado por Byrne (2010). Contudo, Wheaton et al. (1977) sugerem que um valor aproximado ou abaixo de 5 pode ser considerado razoável, bem como Marsh e Hocevar (1985) que indicam o intervalo de 2 a 5 como um ajuste razoável. Além disso, os índices de ajuste incrementais foram iguais a 0,90 (CFI, TLI e IFI). Por sua vez, os índices de ajuste absoluto apresentaram valores abaixo do limite de 0,08 estabelecido pela literatura (HU; BENTLER, 1999; BYRNE, 2010; HAIR et al., 2009), indicando também um bom ajuste do modelo. O RMSEA foi de 0,077. Diante desses índices, conclui-se que o ajuste do modelo proposto é satisfatório. 
Após a verificação do ajuste dos modelos de mensuração e estrutural propostos, foram avaliados os coeficientes estimados para as relações causais entre os construtos (Tabela 4 e Figura 2). A verificação de cada uma das hipóteses da pesquisa foi realizada com a análise da magnitude, direção e significância dos coeficientes padronizados estimados por meio do modelo estrutural, sendo todas as hipóteses não rejeitadas.

Com relação às proporções da variância de cada variável dependente capturadas pelo modelo proposto, foi visto que o modelo é capaz de explicar 75,5\% da variância observada na Atitude, 67,5\% da Intenção de Compra e 69,6\% do Boca a Boca Positivo. Esse resultado indica um bom poder explicativo, mostrando que uma grande proporção da variabilidade das variáveis dependentes de interesse é traduzida pelas variáveis antecedentes empregadas e relações estimadas, superiores aos níveis de explicação obtidos por Amaro e Duarte (2015) para Atitude (62\%) e Intenção de Compra (67\%), mesmo com o modelo proposto neste trabalho sendo mais parcimonioso (com dois construtos e nove hipóteses a menos) do que o estimado por Amaro e Duarte (2015).

Tabela 4. Verificação de relações propostas e coeficientes

\begin{tabular}{l|c|c|c}
\hline Relação proposta & $\begin{array}{c}\text { Coeficiente } \\
\text { padronizado }\end{array}$ & p-valor & $\begin{array}{c}\text { Hipótese } \\
\text { verificada }\end{array}$ \\
\hline Atitude -> Intenção de compra & 0,821 & $<0,001$ & SIM \\
\hline Atitude ->Boca-a-boca positivo & 0,835 & $<0,001$ & SIM \\
\hline Comunicabilidade ->Atitude & 0,391 & $<0,001$ & SIM \\
\hline Complexidade ->Atitude & 0,329 & $<0,001$ & SIM \\
\hline Vantagem relativa ->Atitude & 0,670 & $<0,001$ & SIM \\
\hline Risco percebido ->Atitude & $-0,214$ & $<0,001$ & SIM \\
\hline
\end{tabular}

Fonte: Dados da pesquisa.

Na figura 2, apresentam-se os coeficientes expressos acima das setas de cada relação.

Figura 2. Coeficientes padronizados estimados para o modelo proposto 


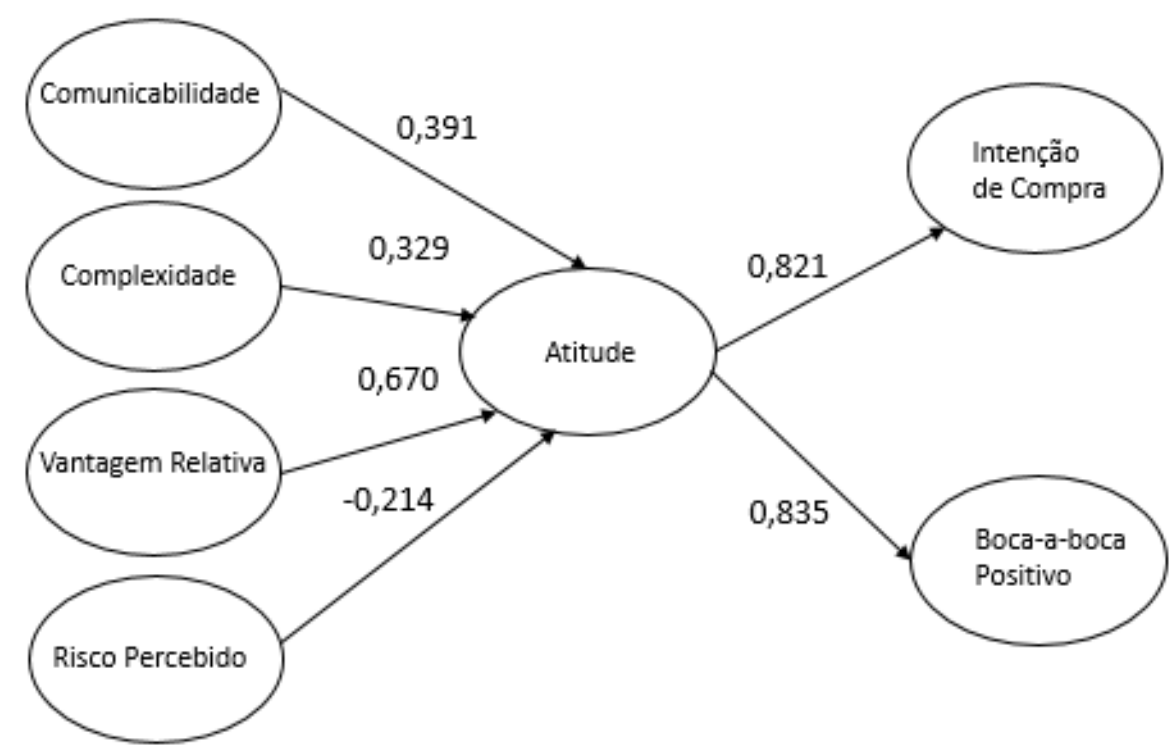

Fonte: Elaboração própria.

\section{Discussão dos resultados}

\subsection{Atitude, intenção de compra e boca a boca positivo}

A confirmação da hipótese 1, indicando uma relação da atitude com a intenção de compra, corrobora com a literatura a respeito de compras online (INGHAM; CADIEUX; MEKKI BERRADA, 2015; VIJAYASARATHY, 2004) e com a de viagens online (AGAG; EL-MASRY, 2016a e b; AMARO; DUARTE, 2015; LEE; QU; KIM, 2007; JENG; LO, 2019).

Considerando o efeito significativo apontado (coeficiente padronizado de 0,821 ), confirma-se a ideia de que a Atitude é determinante para a Intenção de Compra (BIGNÉ et al., 2010; MOROSAN; JEONG, 2008), o que também corrobora com a Teoria da Ação Racionalizada (Theory of Reasoned Action), reforçando o entendimento de que as intenções dos indivíduos resultam da atitude formada a respeito de determinado comportamento (FISHBEIN; AJZEN, 1975).

Já a sustentação da hipótese 2, que apresenta uma relação da atitude com o boca a boca positivo, reforça o entendimento de que a atitude também é um construto relevante para a divulgação de um determinado serviço ou produto (SHIH; LAI; CHENG, 2013). Além disso, a forte relação entre estes construtos (coeficiente padronizado de 0,835) endossa os resultados obtidos por Agag e El-Masry (2016a) que analisaram esta relação sobre a perspectiva do segmento turístico, especificamente sobre comunidade de viagens online. 
ESTUDO DE ANTECEDENTES DA ACEITAÇÃO

DE SITES DE RESERVAS ONLINE, INTENÇÃ̃

DE COMPRA E BOCA A BOCA POSITIVO
Robledo Cunha Rubem Jorge Brantes Ferreira Roberto Pessoa de Queiroz Falcão

Os resultados obtidos indicam, portanto, que assim como a atitude influencia a intenção de compra de viagens online (AMARO; DUARTE, 2015), ela também influencia a intenção de compra por meio de sites de reservas, confirmando que a mesma relação encontrada para a aquisição de viagens pela internet de forma geral também está refletida nas compras relacionadas especificamente aos sites de reservas. Em relação ao boca a boca positivo, da mesma forma que se observou uma influência da atitude sobre a divulgação de comunidades de viagens online (AGAG; EL-MASRY, 2016a), foi observada uma influência significativa da atitude sobre o boca a boca positivo de sites de reservas. Sendo assim, a sustentação dessas hipóteses é importante, pois indica que uma atitude favorável é determinante para que o consumidor venha a comprar ou a divulgar um determinado serviço. Nesse sentido, as características da amostra pesquisada contribuem para este entendimento, visto que há uma presença importante de indivíduos que nunca fizeram qualquer tipo de compras por meio de um site de reserva $(18,95 \%)$ e praticamente metade da amostra não viaja mais que uma vez por ano $(50,67 \%)$.

Esses resultados também sugerem que uma percepção favorável do público gera resultados para as empresas que vão além da própria compra, em linha com as tendências vivenciadas na atualidade (MELIÁN-ALZOLA; FERNÁNDEZ-MONROY; HIDALGO-PEÑATE, 2020). Um indivíduo que não tem condições de viajar, por falta de recursos financeiros ou de tempo, possivelmente não chegará a comprar viagens por meio de sites de reservas. Contudo, se no seu círculo social houver alguém que tenha condições de viajar, é possível que essa pessoa recomende o uso de um site de reserva, mesmo não sendo usuária, dada sua atitude em relação ao serviço.

\subsection{Comunicabilidade}

A confirmação da hipótese 3 traz uma contribuição muito importante para a análise da Comunicabilidade na literatura, visto que não existe ainda um consenso sobre a relevância deste construto. Neste estudo, a Comunicabilidade é tratada como uma diferente forma de influência social (AMARO; DUARTE, 2015).

Nos resultados obtidos para as normas subjetivas, não se tem encontrado suporte para evidenciar uma influência sobre comportamentos relacionados a compras online (PAVLOU; FYGENSON, 2006; WANG et al., 2007) e compras de viagens online (SAN MARTÍN; HERRERO, 2012; JENG; LO, 2019). Já nesse estudo, a comunicabilidade apresentou impactos na formação da atitude relacionada a sites de reservas online. Quanto ao efeito da Comunicabilidade sobre a Intenção de Compra, havia uma certa indefinição sobre a sua contribuição, dado que Morrison et al. (2001) encontram suporte para tal relação 
ESTUDO DE ANTECEDENTES DA ACEITAÇÃO

DE SITES DE RESERVAS ONLINE, INTENÇÃO

DE COMPRA E BOCA A BOCA POSITIVO
Robledo Cunha Rubem Jorge Brantes Ferreira Roberto Pessoa de Queiroz Falcão

em seu estudo, mas, por outro lado, Amaro e Duarte (2015) não observaram esse efeito na aplicação de seu respectivo modelo.

Considerando os resultados para o construto Comunicabilidade, pode-se afirmar que ele tem maior relevância quando relacionado com a atitude, corroborando com Amaro e Duarte (2015), que não encontraram suporte para hipótese que liga esse construto à intenção. Isso também está de acordo com o trabalho seminal de Burnkrant e Cousineau (1975), pois, segundo eles, a influência de outros é o fator que impacta mais profundamente o comportamento de um indivíduo. Os indivíduos se sentem mais seguros quando veem que outros já experimentaram uma nova tecnologia e não sofreram perdas ou desapontamento.

Partindo do pressuposto que o consumo de viagens não é algo restrito a um determinado grupo social; existem consumidores que viajam para perto, outras para longe; algumas com mais idade, outras com menos idade; alguns a trabalho e outros a lazer. Enfim, os perfis e objetivos dos viajantes são muito variados. Nesse sentido, quanto mais perfis e viajantes individuais os sites de reservas conseguem alcançar, mais abrangente se torna o seu público e mais natural se torna o uso desse tipo de serviço (MELIÁN-ALZOLA; FERNÁNDEZ-MONROY; HIDALGO-PEÑATE, 2020).

\subsection{Complexidade}

Verificou-se uma influência da Complexidade sobre a Atitude, porém, diferentemente do que foi previsto inicialmente, esta relação foi positiva. Essa diferença no resultado pode ser explicada em razão da escala utilizada que possuía dois itens relacionados à dificuldade de se utilizar sites de reservas online e outros dois voltados para a facilidade de utilização percebida em relação a este serviço. Somase a isso o fato de que um desses quatro itens (CPL2) foi removido da análise por ter sido identificada uma potencial causa de não conformidade dos índices de ajuste, sendo este justamente um item relacionado à dificuldade de utilização. Dessa forma, entende-se que a escala de complexidade foi compreendida como facilidade de uso pelos participantes da pesquisa, o que é perfeitamente aceitável, dado que as escalas de medição e a definição de complexidade são similares à facilidade de uso do modelo TAM (VENKATESH et al., 2003).

Sabendo disso, pode-se afirmar que os resultados do teste da hipótese 4 corroboram com o entendimento de que, quando se percebe uma determinada tecnologia como algo simples e fácil de usar, existe uma probabilidade maior de aceitação da mesma (DAVIS; BAGOZZI; WARSHAW, 1989), estando também em linha com o que o modelo TAM indica sobre a facilidade de uso, um antecedente importante para a construção da atitude (DAVIS, 1989). 
ESTUDO DE ANTECEDENTES DA ACEITAÇÃO

DE SITES DE RESERVAS ONLINE, INTENÇÃO

DE COMPRA E BOCA A BOCA POSITIVO
Robledo Cunha Rubem Jorge Brantes Ferreira Roberto Pessoa de Queiroz Falcão

Os resultados obtidos para hipótese 4 , sobre a relação da complexidade com a formação da atitude para sites de reserva online, reforçam o que foi identificado também por Cho e Agrusa (2006). Além disso, esse efeito mostra coerência com o que já foi evidenciado sobre os sites para reserva de hotéis (AGAG; EL-MASRY, 2016b; MOROSAN; JEONG, 2008), compra de viagens online (AMARO; DUARTE, 2015) e participação em comunidades de viagens online (AGAG; EL-MASRY, 2016a), confirmando a importância desse construto.

Uma viagem é cercada de tantos detalhes que, muitas vezes, o viajante percebe que não conseguirá resolver tudo sozinho, sendo este, inclusive, um dos motivos pelos quais alguns procuram por intermediários para fazer as compras relacionadas a viagens (ADEOLA; EVANS, 2019). Nesse sentido, foi possível compreender o quanto a complexidade do uso pode influenciar a percepção de sites de reservas online, pois, dado que uma viagem é algo extremamente sensível para os consumidores, é muito importante que os usuários sintam que este serviço é simples e fácil de usar para que se sintam confiantes de planejar e fazer as compras de suas viagens sozinhos (ALMEIDA; ALMEIDA; MOTA, 2019).

\subsection{Vantagem relativa}

O suporte obtido para a hipótese 5 proporciona mais uma evidência da importância da Vantagem Relativa da teoria de difusão de inovações e também da Utilidade Percebida do modelo de aceitação de tecnologias, dada a semelhança existente entre esses dois conceitos (AMARO; DUARTE, 2015; RIEMENSCHNEIDER; HARDGRAVE; DAVIS, 2002; WU; WANG, 2005). Adicionalmente, considerando que a escolha da utilização da Vantagem Relativa para este estudo, consiste na possibilidade de comparação do serviço analisado e as formas de compra de viagens que existiam anteriormente (KARAHANNA et al., 2002; SHIN, 2010), essa relevância fica ainda mais clara, pois indica maior entrega de benefícios dos sites de reserva online em relação às outras formas de aquisição de viagens.

Essa relação significativa da Vantagem Relativa com a Atitude é coerente com o que tem sido observado na literatura, em especial sobre serviços relacionados a viagens online (AMARO; DUARTE, 2015; AGAG; EL-MASRY, 2016a). Dado que a Vantagem Relativa e Utilidade Percebida são construtos que tratam de aspectos semelhantes, esse resultado também contribui com o que é indicado pelo TAM (BAGOZZI; DAVIS; WARSHAW, 1992). 
ESTUDO DE ANTECEDENTES DA ACEITAÇÃO

DE SITES DE RESERVAS ONLINE, INTENÇÃO

DE COMPRA E BOCA A BOCA POSITIVO
Robledo Cunha Rubem Jorge Brantes Ferreira Roberto Pessoa de Queiroz Falcão

É interessante notar que benefícios como descontos, conveniência e variedade de produtos são bastante relevantes para a construção da Atitude (JENG; LO, 2019). Isso faz sentido dada a natureza do turismo, pois são muitas as questões envolvidas em uma viagem. Os descontos são muito bem-vindos, considerando que muitas pessoas sempre buscam fazer o máximo possível em uma viagem, gastando o mínimo possível. Ao economizar com a hospedagem, por exemplo, o consumidor tem condições de fazer determinado passeio que talvez antes não coubesse no orçamento separado para a viagem. A relevância da vantagem relativa do preço pode estar presente em todas as classes sociais, mesmo aquelas com maior poder aquisitivo, considerando que mais da metade dos respondentes declarou renda familiar acima de $\mathrm{R} \$ 6.000$.

Os muitos detalhes concernentes a uma viagem tomam tempo e o esforço para seu planejamento e compra. Dessa forma, a conveniência ganha importância neste segmento, pois o consumidor busca resolver essas questões da forma mais prática e rápida possível. Assim, o acesso aos sites de reservas se torna mais conveniente, gerando uma percepção positiva do serviço. Associado à conveniência, a variedade de opções também se apresenta como um quesito importante na formação de uma atitude favorável. De fato, poder encontrar diversas opções de hotéis e passagens aéreas em um só lugar, de diferentes empresas, facilita muito a decisão dos consumidores, sendo a comparação de ofertas por meio de um site de reservas simplificada.

\subsection{Risco percebido}

A confirmação da hipótese 6, que evidencia uma relação negativa do Risco Percebido com a Atitude, reforça o que já vem sendo indicado pela literatura. $O$ resultado obtido corrobora com o que foi identificado por Bigné et al. (2010), visto que eles também encontraram suporte para esta relação. Todavia, essa evidência é contrária ao que foi percebido por Amaro e Duarte (2015), que também avaliaram a relação do risco percebido com a atitude, porém sem encontrar suporte para a sua hipótese.

De qualquer forma, esta foi a relação que apresentou o menor coeficiente padronizado dentre todas as hipóteses testadas do modelo proposto. Especificamente sobre Atitude, indica que esta relação deve ser novamente estudada em outras pesquisas para haver maior confirmação empírica dos impactos que podem ser gerados por este construto. Portanto, isso indica que ainda há preocupação com o risco na avaliação do serviço. No entanto, com a popularização da internet, esse efeito vem se reduzindo, diminuindo sua relevância sobre a Atitude. Ademais, muitas viagens envolvem um alto valor monetário e um alto grau de ansiedade e expectativa, o que, em contrapartida, gera também um temor de que algo dê errado. 
ESTUDO DE ANTECEDENTES DA ACEITAÇÃO

DE SITES DE RESERVAS ONLINE, INTENÇÃO

DE COMPRA E BOCA A BOCA POSITIVO
Robledo Cunha Rubem

Jorge Brantes Ferreira

Roberto Pessoa de Queiroz Falcão

\section{Considerações finais}

De forma geral, percebe-se que os resultados obtidos estão coerentes com o que vem sendo apresentado na literatura, a respeito de compras de viagens online e, especificamente, sobre a aceitação e o uso de sites de reservas online.

Do ponto de vista teórico, o estudo contribuiu ao apresentar um modelo parcimonioso, embora com alto grau de explicação para a Intenção de Compra e o Boca a boca Positivo. Ao se comparar o presente estudo com o trabalho de Cho e Agrusa (2006), que avaliaram a aceitação de agências de viagens online, o modelo deste estudo oferece contribuições relativas à adição de outros construtos com a Atitude, como Comunicabilidade e Risco Percebido, não avaliados anteriormente, bem como a relação da Atitude com o Boca a boca Positivo. Já ao se comparar com o modelo proposto por Amaro e Duarte (2015), para a Intenção de Compra de viagens online, este modelo contribui com uma simplificação com um alto nível de explicação das variáveis dependentes. Além disso, ainda contribui adicionalmente ao que foi verificado por Agag e El-Masry (2016a), que observaram uma influência da Atitude sobre o Boca a Boca positivo de comunidades online de viagens, uma vez que este estudo confirmou esta mesma relação sobre sites de reservas online. Vale também ser ressaltado que, dada a heterogeneidade da amostra e sua representatividade em termos de perfis diversos de usuários, o modelo proposto também se mostrou robusto.

Em termos de contribuições gerenciais, o estudo aponta os atributos que podem gerar maior aceitação e utilização de sites de reservas on-line. No mercado turístico, onde o consumo de serviços de viagens depende de uma série de fatores, tais como disponibilidade de tempo e recursos do indivíduo, a relação da atitude com o boca a boca demonstra que uma pessoa não precisa necessariamente ser usuária de um determinado produto ou serviço para divulgar o mesmo para outros (MELIÁN-ALZOLA; FERNÁNDEZ-MONROY; HIDALGO-PEÑATE, 2020). Os atributos antecedentes da atitude como comunicabilidade, complexidade, vantagem relativa ou risco podem também ser cruciais para criação de uma campanha de comunicação voltada aos agentes de viagem online. Sendo a vantagem relativa, o construto que teve maior influência sobre a atitude, descontos, conveniência e variedade de opções, o mesmo pode ser considerado importante para consumidores na formação da atitude em relação aos sites de reservas online. Essa relação pode também explicar sua migração para este tipo de serviço, em detrimento das agências de viagens tradicionais e os canais diretos disponibilizados pelos próprios fornecedores (companhias aéreas, hotéis, locadoras, etc.). A diferenciação da oferta e a análise de custo $\mathrm{x}$ benefício tornam-se impactantes na decisão de consumo e comparação dos concorrentes. Ademais, 
ESTUDO DE ANTECEDENTES DA ACEITAC̃̃̃

DE SITES DE RESERVAS ONLINE, INTENÇÃ̃

DE COMPRA E BOCA A BOCA POSITIVO
Robledo Cunha Rubem Jorge Brantes Ferreira Roberto Pessoa de Queiroz Falcão

comunicabilidade, complexidade e risco percebido também são construtos relevantes que não devem ser ignorados pelas empresas.

Ações de experimentação podem ser uma boa forma de popularizar a utilização dos serviços, a exemplo de uma diária de um hotel grátis (ou com desconto) para gerar utilização do site pela primeira vez ou premiando os que indicarem o site para outros. Quanto mais viva a marca ficar na mente dos consumidores, mais natural ela se torna, portanto, o patrocínio de eventos turísticos e anúncios em programas voltados ao tema também devem ser considerados. No decorrer do uso do site, o usuário poderá verificar sua facilidade de navegar e de realizar compras, o que se torna crucial diante da popularização e diversificação dos públicos que não estejam habituados ou tenham receio em utilizar ferramentas online. Outrossim, diminuir a complexidade poderá gerar maior conveniência e aceitação desses sistemas. Contudo, essa facilidade deve ser percebida em todos os dispositivos, tais como computadores, smartphones e tablets, facilitando a compra pelo site a qualquer hora e em qualquer lugar. Sendo a vantagem relativa o construto mais impactante sobre a atitude, o consumidor, motivado pelo benefício concedido, e pela verificação de que tudo ocorreu da forma correta, passará a ter uma atitude mais favorável e um risco percebido menor em relação a esses sites.

Uma das limitações do estudo relaciona-se à forma como os dados foram coletados. Mesmo com a apresentação de exemplos de sites de reservas no início do questionário, é possível que os respondentes tenham considerado, em suas respostas, os sites de hotéis, companhias aéreas ou de empresas que oferecem atrações turísticas e que disponibilizem reservas em seus sites. Outra possível limitação seriam os efeitos moderadores gerados por variáveis demográficas (como idade e gênero), ou relativas ao nível de experiência dos respondentes com o tipo de serviço avaliado, não consideradas na análise.

Nesse sentido, com a crescente adoção dos sites de reservas, propõe-se estudar efeitos moderadores sobre as relações propostas no modelo (como idade, ou mesmo de pessoas que já viajaram para o exterior e das que não viajaram). Poderiam ser incluídos outros construtos e novas relações no modelo, para avaliar se essas inserções contribuiriam ou não com a explicação para a intenção de compra e boca a boca positivo de sites de reservas online.

\section{Referências}

AAKER, D. A. et al. Marketing Research. 11. Ed., Wiley, 2012.

ADEOLA, O.; EVANS, O. Digital tourism: mobile phones, internet and tourism in Africa. Tourism Recreation Research, v. 44, n. 2, p. 190-202, 2019. 
AGAG, G.; EL-MASRY, A. A. Understanding consumer intention to participate in online travel community and effects on consumer intention to purchase travel online and WOM: an integration of innovation diffusion theory and TAM with trust. Computers in Human Behavior, v. 60, p. 97-111, $2016 \mathrm{a}$.

AGAG, G.; EL-MASRY, A. A. Understanding the determinants of hotel booking intentions and moderating role of habit. International Journal of Hospitality Management, v. 54, p. 52-67, $2016 \mathrm{~b}$.

ALMEIDA, F.; ALMEIDA, J.; MOTA, M. Perceptions and trends of booking online payments in tourism. Journal of Tourism and Services, v. 10, n. 18, p. 1-15, 2019.

AMARO, S.; DUARTE, P. An integrative model of consumers' intentions to purchase travel online. Tourism Management, v. 46, p. 64-79, 2015.

BAEK, H.; AHN, J.; CHOI, Y. Helpfulness of online consumer reviews: readers' objectives and review cues. International Journal of Electronic Commerce, v. 17, n. May 2015, p. 99-126, 2012.

BAGOZZI, R.; DAVIS, F.; WARSHAW, P. Development and test of a theory of technological learning and usage. Human Relations, v. 45, p. 659-686, 1992.

BIGNÉ, E. et al. Why some internet users don't buy air tickets online. In U. Gretzel, R. Law, \& M. Fuchs (Eds.), Information and communication technologies in tourism. Vienna, Austria: Springer. p. 209-221. 2010.

BUHALIS, D.; LAW, R. Progress in information technology and tourism management: 20 years on and 10 years after the Internet-The state of eTourism research. Tourism Management, v. 29, n. 4, p. 609-623, 2008.

BURNKRANT, R. E.; COUSINEAU, A. Informational and normative social influence in buyer behavior. Journal of Consumer Research, v. 2, n. 3, p. 206-215, 1975.

BYRNE, B. M. Structural equation modeling with amos: basic concepts, applications and programming. $2^{\mathrm{a}}$ ed. Routledge, NY, 2010.

CASALÓ, L. V.; FLAVIÁN, C.; GUINALÍU, M. Determinants of the intention to participate in firmhosted online travel communities and effects on consumer behavioral intentions. Tourism Management, v. 31, n. 6, p. 898-911, 2010.

CASTILLO-MANZANO, J. I.; LÓPEZ-VALPUESTA, L. The decline of the traditional travel agent model. Transportation Research Part E: Logistics and Transportation Review, v. 46, n. 5, p. 639649, 2010.

CHEYNE, J.; DOWNES, M.; LEGG, S. Travel agent vs internet: What influences travel consumer choices? Journal of Vacation Marketing, v. 12, n. 1, p. 41-57, 2006.

CHO, Y. C.; AGRUSA, J. Assessing use acceptance and satisfaction toward online travel agencies. Information Technology \& Tourism, v. 8, n. 3, p. 179-195, 2006.

DAVIS, F. D. Perceived usefulness, perceived easeof use, and user acceptance of information technology. MIS Quarterly, v. 13, n. 3, p. 319-340, 1989.

Observatório de Inovação do Turismo - Revista Acadêmica

Vol. XIX, n ${ }^{\circ}$, dezembro - 2020 
DAVIS, F. D.; BAGOZZI, R.; WARSHAW, P. User acceptance of computer technology: a comparison of two theoretical models. Management Science, v.35, n. 8, p. 982-1003, 1989.

DICKINGER, A.; MAZANEC, J. Consumers' preferred criteria for hotel online booking. Information and Communication Technologies in Tourism 2008, p. 244-254, 2008.

EUROMONITOR INTERNATIONAL. Online Travel Sales to Residents in Brazil Country Report. Sep 2017. Disponível em: https://www.euromonitor.com/online-travel-sales-to-residents-inbrazil/report. Acesso em: 14 mai. 2020.

EUROMONITOR INTERNATIONAL. What the Future Holds for Travel in 2020. Dec 2019. Disponível em: https://blog.euromonitor.com/what-the-future-holds-for-travel-in-2020/. Acesso em: 14 mai. 2020.

FORNELL, Claes; LARCKER, David F. Evaluating structural equation models with unobservable variables and measurement error. Journal of marketing research, v. 18, n. 1, p. 39-50, 1981.

FISHBEIN, M.; AJZEN, I. Belief, atitude. intention and behaviour: an introduction to theory and research. Reading, MA Addison-Wesley Publishing, 1975.

GODES, D., MAYZLIN, D. Using online conversations to study word-of-mouth communication. Marketing science, v. 23, n. 4, p. 545-560, 2004.

GRETZEL, U.; FESENMAIER, D. R. Information technology: shaping the past, present, and future of tourism. In T. Jamal, \& M. Robinson (Eds.). The SAGE handbook of tourism studies. London: Sage. pp. 558-580, 2009.

HAIR, J. F. et al. Multivairate Data Analisys. 7. ed. Upper Saddle River: Prentice-Hall, 2009.

HENNIG-THURAU, T. et al. Electronic word-of-mouth via consumer-opinion platforms: what motivates consumers to articulate themselves on the Internet? Journal of Interactive Marketing, vol. 18, n.o 1, p. 38-52, 2004.

HO, C. I.; LIN, M. H.; CHEN, H. M. Web users' behavioural patterns of tourism information search: From online to offline. Tourism Management, v. 33, n. 6, p. 1468-1482, 2012.

HU, L. T.; BENTLER, P. M. Cutoff criteria for fit indexes in covariance structure analysis: Conventional criteria versus new alternatives. Structural Equation Modeling, v. 6, n. 1, p. 1-55, 1999.

INGHAM, J.; CADIEUX, J.; MEKKI BERRADA, A. E-Shopping acceptance: a qualitative and metaanalytic review. Information and Management, v. 52, n. 1, p. 44-60, 2015.

JARVENPAA, S. L.; TODD, P. A. Is there a future for retailing on the Internet. Electronic marketing and the consumer, v. 1, n. 12, p. 139-154, 1997.

JENG, S.-P.; LO, M.-F. Lowest price guarantees on airline websites: Perceived believability, perceived value, and purchase intentions. Journal of Air Transport Management, v. 75, p. 85-91, 2019. 
JENSEN, J. M. Shopping orientation and online travel shopping: the role of travel experience.

International Journal of Tourism Research, v. 14, n. 1, p. 56-70, 2012.

KARAHANNA, E. et al. Individual differences and relative advantage: The case of GSS. Decision Support Systems, v. 32, n. 4, p. 327-341, 2002.

KIM, L. H.; KIM, D. J.; LEONG, J. K. The effect of perceived risk on purchase intention in purchasing airline tickets online. Journal of Hospitality \& Leisure Marketing, v.13, n. 2, p. 33-53, 2005.

KIM, L. H.; QU, H.; KIM, D. J. A Study of perceived risk and risk reduction of purchasing air- tickets online. Journal of Travel \& Tourism Marketing, v. 26, n. 3, p. 203-224, 2009.

KOLSAKER, A.; LEEKELLEY, L.; CHOY, P. C. The reluctant Hong Kong consumer: purchasing travel online. International Journal of Consumer Studies, v. 28, n. 3, p. 295-304, 2004.

LADHARI, R.; MICHAUD, M. EWOM. Effects on hotel booking intentions, attitudes, trust, and website perceptions. International Journal of Hospitality Management, v. 46, p. 36-45, 2015.

LAW, R.; CHAN, I. C. C.; WANG, L. A comprehensive review of mobile technology use in hospitality and tourism. Journal of Hospitality Marketing \& Management, v. 27, n. 6, p. 626-648, 2018.

LEE, H. Y.; QU, H.; KIM, Y. S. A study of the impact of personal innovativeness on online travel shopping behavior - A case study of Korean travelers. Tourism Management, v. 28, n. 3, p. 886-897, 2007.

LEUNG, D. et al. Social media in tourism and hospitality: a literature review. Journal of Travel and Tourism Marketing, v. 30, n. 1-2, p. 3-22. 2013.

LIEN, C. H.; CAO, Y. Examining WeChat users' motivations, trust, attitudes, and positive word-ofmouth: evidence from China. Computers in Human Behavior, v. 41, p. 104-111, 2014.

LIN, P. J.; JONES, E.; WESTWOOD, S. Perceived risk and risk-relievers in online travel purchase intentions. Journal of Hospitality and Leisure Marketing, v. 18, n. 8, p. 782-810, 2009.

LITVIN, S. W.; GOLDSMITH, R. E.; PAN, B. Electronic word-of-mouth in hospitality and tourism management. Tourism Management, v. 29, n. 3, p. 458-468, 2008.

LIU, Z.; PARK, S. What makes a useful on-line review? Implication for travel product websites.

Tourism Management, v. 47, p. 140-151, 2015.

LÓPEZ, M.; SICILIA, M. Determinants of E-WOM influence: the role of consumers' internet experience. Journal of theoretical and applied electronic commerce research, v. 9, n. 1, p. 28-43, 2014.

LUO, Q.; ZHONG, D. Using social network analysis to explain communication characteristics of travel-related electronic word-of-mouth on social networking sites. Tourism Management, v. 46, p. 274-282, 2015. 
MARSH, H. W.; HOCEVAR, D. Application of confirmatory factor analysis to the study of selfconcept: first- and higher order factor models and their invariance across groups. Psychological Bulletin, v. 97, n. 3, p. 562-582, 1985.

MELIÁN-ALZOLA, L.; FERNÁNDEZ-MONROY, M.; HIDALGO-PEÑATE, M. Tourism management perspectives. Tourism Management, v. 33, p. 100606, 2020.

MOROSAN, C.; JEONG, M. Users' perceptions of two types of hotel reservation Web sites. International Journal of Hospitality Management, v. 27, n. 2, p. 284-292, 2008.

MORRISON, A. M. et al. Predicting usage of the internet for travel bookings. Information Technology \& Tourism, v. 4, n. 1, p. 15-30, 2001.

PAN, Y.; ZHANG, J. Q. Born unequal: a study of the helpfulness of user generated product reviews. Journal of Retailing, v. 87, n. 4, p. 598-612, 2011.

PAVLOU, P. A.; FYGENSON, M. Understanding and predicting electronic commerce adoption: An extension of the theory of planned behavior. MIS Quarterly, v. 30, n. 1, p. 115-143, 2006.

PODSAKOFF, P. M.; ORGAN, D. W. Self-reports in organizational research: problems and prospects. Journal of management, v. 12, n. 4, p. 531-544, 1986.

QU, H.; LEE, H. Travelers' social identification and membership behaviors in online travel community. Tourism Management, v. 32, n. 6, p. 1262-1270, 2011.

RIEMENSCHNEIDER, C. K.; HARDGRAVE, B. C.; DAVIS, F. D. Explaining software developer acceptance of methodologies: a comparison of five theoretical models. IEEE Transactions on Software Engineering, v. 28, n. 12, p. 1135-1145, 2002.

ROGERS, E. Diffusion of innovations. 5 ed. New York: Free Press, 2003.

SAN MARTÍN, H.; HERRERO, Á. Influence of the user's psychological factors on the online purchase intention in rural tourism: integrating innovativeness to the UTAUT framework. Tourism Management, v. 33, n. 2, p. 341-350, 2012.

SCHREIBER, J. B. et al. Reporting structural equation modeling and confirmatory factor analysis results: a review. Journal of Educational Research, v. 99, n. 6, p. 323-337, 2006.

SHIH, H.; LAI, K.; CHENG, T. C. E. Informational and relational influences on electronic word of mouth: an empirical study of an online consumer discussion Forum. International Journal of Electronic Commerce, v. 17, n. 4, p. 137-166, 2013.

SILVA, M. M.; MENDES FILHO, L.; MARQUES JUNIOR, S. Comentários de Viajes Online sobre medios de Hospedaje: un abordaje cuantitativo con viajeros de la ciudad de Natal (Brasil). Estudios y Perspectivas en Turismo, v. 28, p. 185-206, 2019.

SILVA, D. S.; MENDES-FILHO, L.; CORREA, C. H. W. Comentários de Viagem na Internet: Fatores que influenciam a intenção de escolha de um destino de viagem. Pasos (EI Sauzal), v. 15, p. 229-244, 2017.

Observatório de Inovação do Turismo - Revista Acadêmica

Vol. XIX, n ${ }^{\circ}$, dezembro - 2020 
SPERBER, A. D. Translation and validation of study instrument for cross-cultural research.

Gastroenterology, v. 126, n. 1, p. 124-128, 2004.

VENKATESH, V. et al. User acceptance of information technology: toward a unified view. MIS Quarterly, v.27, n. 3, pp. 425-478, Setembro 2003.

VERMEULEN, I. E.; SEEGERS, D. Tried and tested: the impact of online hotel reviews on consumer consideration. Tourism Management, v. 30, n. 1, p. 123-127, 2009.

VIJAYASARATHY, L. R. Predicting consumer intentions to use on-line shopping: the case for an augmented technology acceptance model. Information and Management, v. 41, n. 6, p. 747-762, 2004.

WANG, M. S. et al. Effects of online shopping attitudes, subjective norms and control beliefs on online shopping intentions: a test of the theory of planned behaviour. International Journal of Management, v.24, n.2, p. 296-302, 2007.

WANG, Y.; FESENMAIER, D. R. Towards understanding members' general participation in and active contribution to an online travel community. Tourism Management, v. 25, n. 6, p. 709-722, 2004.

WANG, Y. et al. Understanding travellers' preferences for different types of trip destination based on mobile internet usage data. Transportation Research Part C: Emerging Technologies, v. 90, p. 247-259, 2018.

WESTBROOK, R. A. Product/consumption-based affective responses and postpurchase processes. Journal of Marketing Research, v. 24, n. 3, p. 258-270, 1987.

WHEATON, B. et al. Assessing reliability and stability. Sociological Methodology. v. 8, p. 84-136, 1977.

WU, J. H.; WANG, S. C. What drives mobile commerce? An empirical evaluation of the revised technology acceptance model. Information and Management, v. 42, n. 5, p. 719-729, 2005.

WU, J. J.; CHANG, Y. S. Effect of transaction trust on e-commerce relationships between travel agencies. Tourism Management, v. 27, n. 6, p. 1253-1261, 2006.

YE, Q. et al. The influence of user-generated content on traveler behavior: An empirical investigation on the effects of e-word-of-mouth to hotel online bookings. Computers in Human Behavior, v. 27, n. 2, p. 634-639, 2011.

YU, S. F. Price perception of online airline ticket shoppers. Journal of Air Transport Management, v. 14, n. 2, p. 66-69, 2008. 